\title{
Spinal Cord Compression, a Rare Neurofibromatosis Complication
}

\section{Habib Abdoul Karim Ouiminga ${ }^{*}$, Sylvain D. Zabsonré2, Aminath Kélani ${ }^{3}$, Souleymane Ouattara ${ }^{4}$, Assane Dravé5, Raphael Marie Kabore ${ }^{6}$, Désiré Harouna Sankara7, Magatte Gaye ${ }^{8}$}

\author{
${ }^{1}$ Department of Neurosurgery, CHU Tengandogo, Ouagadougou, Burkina Faso \\ ${ }^{2}$ Department of Neurosurgery, CHU Yalgado Ouédraogo, Ouagadougou, Burkina Faso \\ ${ }^{3}$ Department of Neurosurgery, Hopital National de Niamey, Niger \\ ${ }^{4}$ Department of Pathology, CHU Tengandogo, Ouagadougou, Burkina Faso \\ ${ }^{5}$ Department of Neurology, CHU Ouahigouya, Ouahigouya, Burkina Faso \\ ${ }^{6}$ Department of Neurology, CHU Tengandogo, Ouagadougou, Burkina Faso \\ ${ }^{7}$ Department of Imagery, CHU Tengandogo, Ouagadougou, Burkina Faso \\ ${ }^{8}$ Department of Neurosurgery, Hôpital Général Grand Yoff, Dakar, Senegal \\ Email: *ouimkar@gmail.com
}

How to cite this paper: Ouiminga, H.A.K., Zabsonré, S.D., Kélani, A., Ouattara, S., Dravé, A., Kabore, R.M., Sankara, D.H. and Gaye, M. (2019) Spinal Cord Compression, a Rare Neurofibromatosis Complication. World Journal of Neuroscience, 9, 191-198. https://doi.org/10.4236/wjns.2019.93012

Received: June 3, 2019

Accepted: August 10, 2019

Published: August 13, 2019

Copyright $\odot 2019$ by author(s) and Scientific Research Publishing Inc. This work is licensed under the Creative Commons Attribution International License (CC BY 4.0).

http://creativecommons.org/licenses/by/4.0/

\begin{abstract}
Objective: The objective of this study is to report a case of spinal cord compression, which is a rare complication of neurofibromatosis type 1. Observation: We report the case of a 45 -year-old man, which presented a syndrome of thoracic spinal cord compression at the stage of spastic paraparesis. Its installation was gradually over 6 months associated with the inaugural back pain. He had a clinical history of neurofibromatosis type 1 with "Café-au-lait" spots. There were multiple painless nodules under the skin of different size on the chest, forearms and legs. A large isolated nodule, purplish was observed on the chest. The neuro-imaging showed a para-spinal anterior mass expansion inside the spinal canal causing spinal compression at the level of the second and third thoracic vertebra. It extends into the intervertebral foramen of the third and fourth thoracic vertebra leading to a scalloping. A second large heterogeneous left intra-abdominal mass containing cyst areas and calcifications was discovered in imaging. After a spinal decompression with laminectomy of the second and third thoracic vertebra, the reduction of pain and motor recovery was gradual. The large nodule excision was performed. Histology found a plexiform neurofibroma. Excision of the left intra-abdominal mass could not be performed because the patient's consent had not been obtained. Conclusion: The spinal cord compression is a rare complication of neurofibromatosis type 1 . However, it is essential to think about it in front of any spinal cord symptoms or any atypical long term spinal pain.
\end{abstract}




\section{Keywords}

Neurofibromatosis, Von Recklinghausen's Disease, Spinal Cord Compression

\section{Introduction}

Type 1 neurofibromatosis or Von Recklinghausen disease is a benign congenital multi-systemic pathology with mainly neuro-cutaneous tropism [1] [2] [3]. It is an autosomal dominant tumor most readily characterized by the development of multiple neurofibromas of the peripheral nerves [2] [4]. Neurofibromas can grow along with the spinal nervous and in less than $1 \%$ of the cases, they lead to a spinal compression [2] [4]. MRI finds its application in the diagnosis of this complication and spreading assessment. It requires a decompressive surgery without delay because it involves the functional prognosis. The objective of this study is to report a case of thoracic spinal cord compression, which is a rare complication of neurofibromatosis type 1 .

\section{Clinical Observation}

We report the case of a farmer, 45-year-old male patient, who consulted for a syndrome of spinal cord compression with spastic paraparesis. Its installation was progressive in 6 months. The patient had a clinical history of neurofibromatosis type 1 known with "Café-au-lait spots" since his early childhood. No first-degree relatives, (siblings and a paternal ascendant) were suffering from neurofibromatosis type 1 . The muscular strength was quoted as 2 to the left and 3 to the right on a scale of 5 . The high back pain was inaugural with thoracic irradiation in hemi-belt. A hypertonia of the lower limbs was observed. There was a discreet thoracic scoliotic attitude.

Small multiple cutaneous neurofibromas, of pink-purplish color, with soft consistency, were present on the forearms, thorax (Figure 1(A) and Figure 1(B)), neck, back and legs. A large skin nodule measuring 4 on $3 \mathrm{~cm}$, painless, purplish color was observed on the chest (Figure 1). There were the "Café-au-lait" spots (Figure $1(C)$ ), and extended melano-dermal macules, sitting on the thorax and abdomen (Figure 1(D)). These skin lesions would allow the diagnosis of Neuro-Fibromatosis type 1 according to the criteria of the National Institutes of Health (Table 1).

MRI of the thoracic spine showed an anterior thoracic para-spinal mass next to the homogeneous, osteolytic lesion was hyperintense signal in T2-weighted. It was $101 \mathrm{~mm}$ in height and $20 \mathrm{~mm}$ (Figure 2(A)) and it enhances on the periphery after Gadolinium injection (Figure 2(C)). Its extension was inside the spinal canal. The spinal cord was compressed at the level of the second and third thoracic vertebra (Figure $2(B)$ ). Abdominal MRI showed a large intra-abdominal mass tissue on the left estimated at $170 \times 150 \times 100 \mathrm{~mm}$, heterogeneous containing cyst and calcifications (Figure 2(D)). The mass was hyper intense signal 

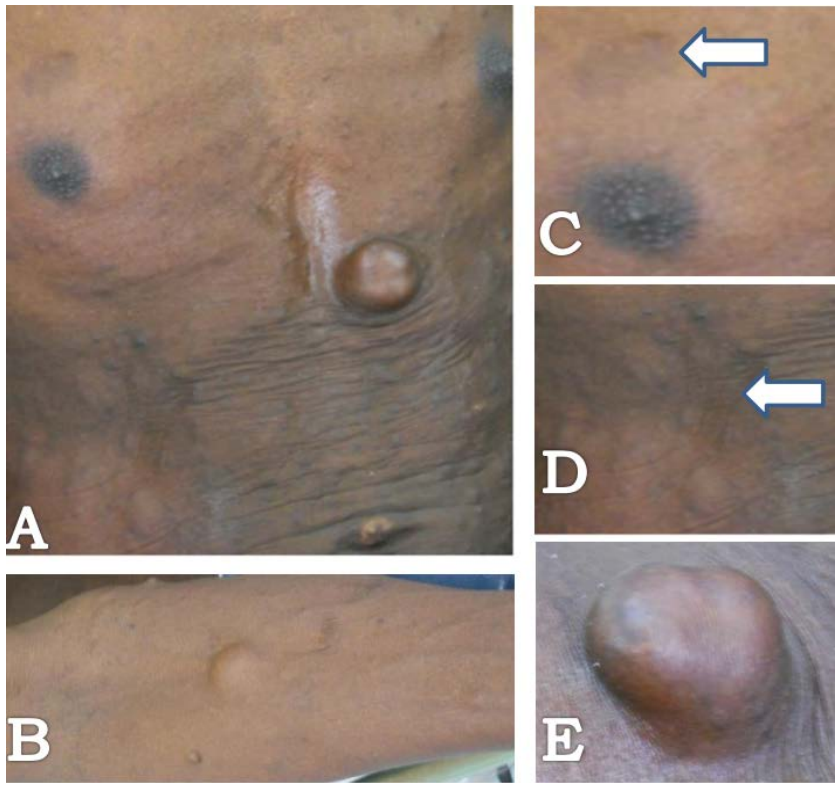

Figure 1. Cutaneous neurofibroma of the chest (A), cutaneous neurofibroma of the forearm (B). "Café-au-lait" spots (C), melano-dermal macules, sitting on the chest (D). neurofibroma, purplish, subcutaneous large pedunculated (E).

Table 1. Clinical diagnostic criteria for neurofibromatosis type 1 of the National Institutes of Health [5].

Diagnostic criteria of Neurofibromatosis Type 1

Diagnostics used in the presence of $\geq 2$ clinical criteria

At least six café-au-lait macules

$(>5 \mathrm{~mm}$ diameter in prepubertal individuals

and $>15 \mathrm{~mm}$ in postpubertal individuals)

At least two neurofibromas of any type, or one

plexiform neurofibroma

Freckling in axillary or inguinal regions

1 Opticglioma

At least two Lisch nodules (iris hamartomas)

A distinctive osseous lesion

(sphenoid dysplasia or tibial pseudarthrosis)

A first degree relative with NF1

in T2-weighted. It was hypointense signal in T1-weighted, with no enhancement after gadolinium. Bone lesions were highlighted using spinal CT. It extends in the intervertebral foramen of the third and fourth thoracic vertebra making a scalloping (Figure 3(A)). There is a discreet thoracic scoliosis (Figure 3(B)).

We had opted for a spinal decompression with laminectomy of the second and third thoracic vertebra associated with biopsy of the lesion. The excision of the large skin nodule was performed. Afunctional and motor rehabilitation completed the treatment. Excision of the left intra-abdominal mass could not be performed because the patient's consent had not been obtained. The discreet thoracic scoliosis was controlled by functional rehabilitation methods and tho- 
racic contention.

The histology of the nodule and the biopsy confirmed the diagnosis by showing an aspect of plexiform neurofibroma (Figure 4) with Schwann cells, fibroblasts

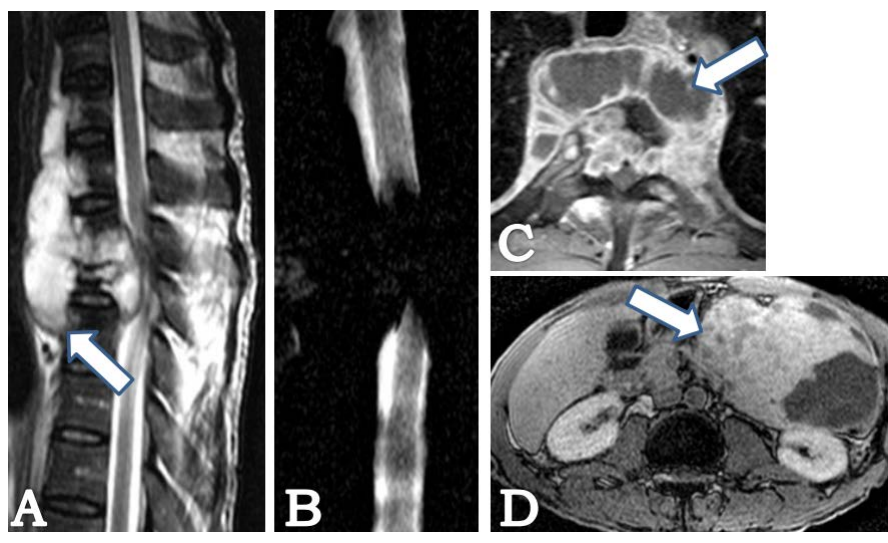

Figure 2. Anterior thoracic para-vertebra mass in sagittal T2-weighted (A), Compression of the spinal cord (B), Contrast of the mass shooting in cup axial (C), large intra-abdominal mass tissue on the left containing cyst and calcifications (D).

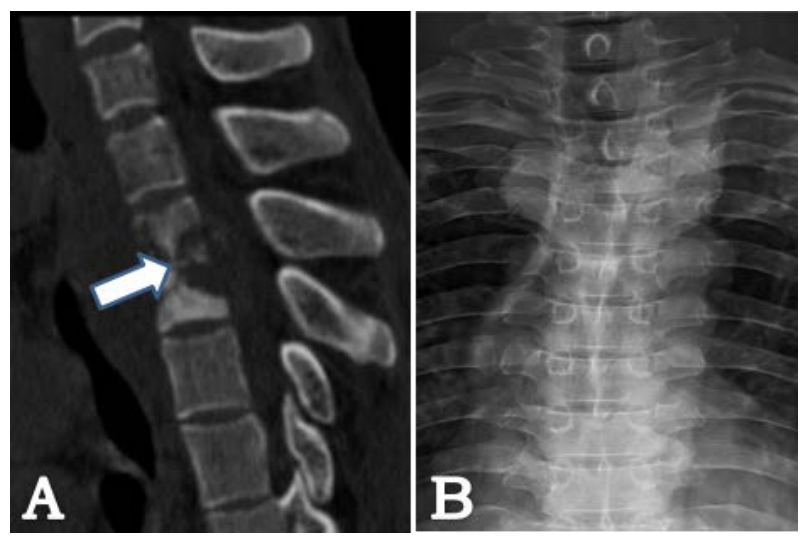

Figure 3. The standard $\mathrm{x}$-ray showed: Thoracic vertebra scalloping (A), discreet thoracic scoliosis (B).

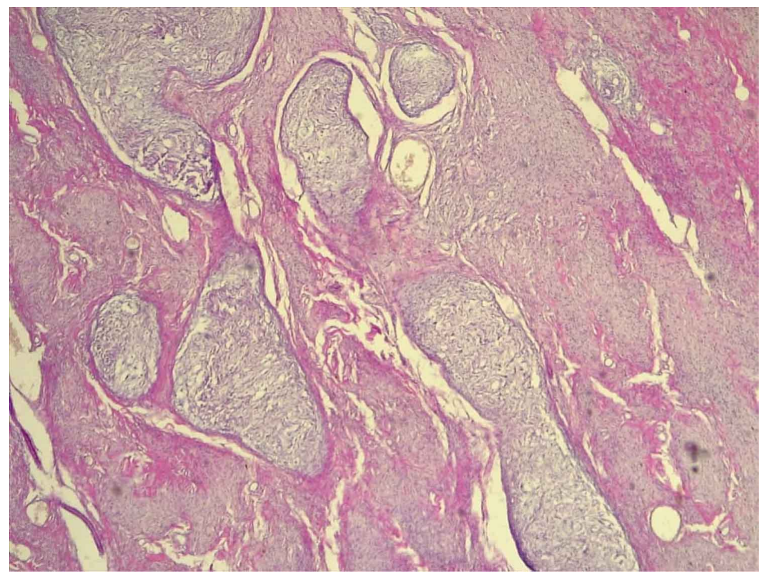

Figure 4. Histological aspects of plexiform neurofibroma. (Hematoxylin Eosin, original magnification $\times 4$ ). 
and many perineural cells, endothelial, and mast cells. The reduction of pain and motor recovery was gradual. After one year of follow up, the walking was autonomous with paresthesia, some residual back pain and spasticity controlled by DANTROLENE. The consent of the patient was still not obtained for ablation of intra-abdominal mass. Then he was lost from view.

\section{Discussion}

Intra-spinal neurofibroma is a rare complication of neurofibromatosis type I. In less than $1 \%$ of cases it leads to a spinal compression [1] [3] [4]. This is the first case of an adult identified in our practice. Neurofibromatosis type 1 corresponds to the classical form described by Von Recklinghausen in 1882. Its incidence is estimated at 1 for 3000 births [1] [2] [3]. Adult is mostly concerned after 40 years [2] [4]. It affects both sexes [2] [4]. It is a genetic disease with autosomal dominant transmission. It is due to a mutation of the tumor suppressor gene located on chromosome 17q11.2 [2] [5] [6]. The protein encoded by this gene is the neurofibromin which intervenes in the control of differentiation and cell proliferation inhibiting the way of activation of $\mathrm{p} 21$ ras. It is a high penetration disease, $80 \%$ of which is of paternal origin [2]. Novo mutations represent about half of the cases [7]. This theory could apply in our case. The presence of neurofibromatosis on one first degree relative was not found in the progeny (in a paternal ascendant or in siblings). Skin lesions present since the childhood could be explained by the neuro-ectodermal involvement which would also be at the origin of neurological lesions [1] [6] [8]. The development and the extension of plexiform neurofibroma inside the spinal canal would explain spinal compression [1] [3] [7]. Its clinical expression, in our case, is that of a progressive spinal cord compression, as reported by the authors [6] [9]. The dorsal pain irradiated as intercostal neuralgia precedes the progressive onset of a neurological deficit of the lower limbs. Subjective sensory disorders such as paresthesia and dysesthesia are also described [2] [9]. One of the essential clinical elements is the length of their evolution. It can reach several months before the diagnosis, as described in our practice or even years [8] [9]. These are tumors usually slow growing "Hourglass" toward the inside of the chest. They remain well tolerated for a long time, and can reach a considerable size [2] [5] [9]. This fact could explain the slow progression of the signs that we had described. The deformation in scoliosis although discrete in our case corresponds to vertebral dysplasia also reported in the literature to varying degrees in $10 \%$ to $30 \%$ of cases [10].

These various signs are generally preceded by cutaneous manifestations [2] [8] [9] which are in the foreground. The multiple small subcutaneous nodules we have described are similar to the cutaneous neurofibromas described in the literature [2] [9]. These are benign tumors that occur only at puberty and $95 \%$ of adults with neurofibromatosis type 1 carry them [2]. The larger one had the clinical characteristics of a plexiform neurofibroma commonly described: large isolated nodule, purplish, soft, and generally not painful [9] [11]. The extensive mela- 
no-dermal areas described in our case, have the same meaning as "Café-au-lait" spots [4] [11]. Although much less common, they can also contribute to the diagnosis of neurofibromatosis type 1 which remains clinical with well-defined criteria [4] [5] [10]. In case of neurologic manifestations as observed in our case, the imagery becomes an indispensable tool for the diagnosis in order to discuss the possibilities of surgical resection of the responsible lesion [6].

The MRI was the essential diagnostic tool [1] [7] [8]. It performed well for our patient by showing the commonly described the suffering of spinal cord, resulting from the mass effect and a prolonged spinal cord compression [3] [8]. The characteristics of the tumor at causing this spinal cord pain were also reported in published reviews [1] [3] [7]. It is homogeneous more or less voluminous and appears in hyper intense signal in T2-weighted. The extension can be done in intervertebral foramen, intradural or extradural [1] [7]. But visceral manifestations are also described including mediastinal, abdominal neurofibromas, likely to malignant transformation [1] [11]. They are heterogeneous with a cystic appearance and identical calcifications as described in our experience.

In neurofibromatosis type 1, CT is less effective than MRI [6] in the diagnosis of spinal compression. It perfectly shows the bone lesions such as the scalloping which is very evocative [2] [4] [10] [11] that we have described. The vertebral dysplasia, causing scoliosis observed in our case, is the most common bone complications [2] [10]. It may require surgery in $37 \%$ of cases [2] [10]. Monitoring is necessary to detect bone's deformities [4] [10]. MRI makes differential diagnoses, with the POTT disease that is frequent in our endemic areas. However, it gives no specific element. It is an essential therapeutic guidance tool allowing a better surgical approach [7] [8].

Complete resection of the tumor is recommended by the authors [3]. However, it is not always possible. In our study, the aim of the surgery was the spinal decompression and to get a histological diagnosis. Our technical tray was limited; a laminectomy was a safe option. An extensive thoracotomy tumor resection required thoracic spine stabilization [3] [10], because the thoracic spine was weakened by the scalloping. The thoraco-lumbar rigid corset permitted spine stabilization associated with physiotherapy as described in the literature [9] [10].

Regardless of the therapeutic option, it can only be a palliative treatment because there is no specific treatment for the disease [4] [9]. The dermatological manifestations can constitute an aesthetic discomfort with an alteration of the quality of life [4] [9]. Plastic and reconstructive surgery could be proposed. The goal of plexiform neurofibroma surgery is often debulking [4] [9]. In our case, only the large skin nodule had been removed by this technique. The other neurofibromas being small and scattered had been monitored only. Other therapeutic options are proposed including $\mathrm{CO}_{2}$ laser therapy for skin forms, dermatologic surgery for plexiform lesions [4] [9]. Recent clinical trials involving tyrosine kinase inhibitor have shown a decrease in tumor volume by greater than 
$20 \%$ [9].

The functional prognosis is generally good after a decompressive surgery [3]. Only an early diagnosis and surgical management allow for an optimal functional outcome [8]. An early post-surgery MRI is recommended. It allows to evaluate the existence or not of a residue, the quality of the decompression [4]. The definitive diagnosis can only be established with the neuro-pathological confirmation [6] [11] [12]. It remains the only one diagnostic confirmation exam. The conclusions of the histological examination we described were similar to those reported by the authors [6] [9] [11] [12]. Schwann cells, fibroblasts and many perineural cells, endothelial and melanin cells were characteristics of plexiform neurofibroma [11] [12]. It is benign tumors [11] [12]. However morbidity and mortality related to neurofibromatosis type 1 result from the occurrence of multisystem complications [1]. The monitoring of progressive lesions by the imagery is important to detect them [6] [7]. Malignant degeneration is the main complication due to their severity frequency around $3 \%-4 \%$ of cases [1]. This malignant degeneration is responsible for $60 \%$ of the causes of death [1]. A surgical biopsy should be performed when there is the appearance of predictive signs of malignant transformation. It results in a rapid increase in size of a neurofibroma, the appearance or modification of pain [1]. The estimated life expectancy is 54 years on average [2].

\section{Conclusion}

The spinal cord compression is a rare complication of neurofibromatosis type 1 . However, it is essential to think about this in front of any long term spinal cord symptoms or atypical spinal pain occurring in a context of neurofibromatosis type 1 , given the major risk of disabilities. Neurofibromatosis type 1 can be accompanied by complications that we can avoid through early detection and clinical follow-up, in the absence of curative treatment.

\section{Informed Consent}

Written informed consent was obtained from the patient for publication of this manuscript and any accompanying images

\section{Conflicts of Interest}

The authors have no conflicts of interest to declare.

\section{References}

[1] Bile-Gui, L.N., Diabate, A.S., Dede, N.S., Kabas, R.M., Kouadio, E., Ohui-Acko, E., et al. (2015) MRI Pattern of Cervical Spinal Cord Compression by Plexiform Neurofibromas: A Case Report. Journal African D'imagerie Médicale, 3, 178-185.

[2] Kresak, J.L. and Walsh, M. (2016) Neurofibromatosis: A Review of NF1, NF2, and Schwannomatosis. Journal of Pediatric Genetics, 5, 98-104. https://doi.org/10.1055/s-0036-1579766

[3] Michel, P., Finet, P. and Raftopoulos, C. (2017) Myelopathy Secondary to a Spinal 
Cord Compression Induced by C2 Nerve Tumors during Head Rotation. Neurochirurgie, 63, 62. https://doi.org/10.1016/j.neuchi.2016.11.091

[4] Valeyrie-allanore, L. (2010) Neurofibromatosis 1: Complications in Adulthood, Management and Treatment. Neurologie, 2, 248-251.

[5] (1988) National Institutes of Health Consensus Development Conference Statement: Neurofibromatosis. Bethesda, Md., USA, July 13-15, 1987. Neurofibromatosis, 1, 172-178.

[6] Brosius, S. (2010) A History of von Recklinghausen's NF1. Journal of the History of the Neurosciences, 19, 333-348. https://doi.org/10.1080/09647041003642885

[7] Aidara, C.M., Diop, D., Ndiaye, O.K., Diop, A.D., Ndiaye, M.A., Diop, A.N., et al. (2015) Contribution of Imaging in Neurofibromatosis Type 1. Feuillets de Radiologie, 55, 31-36. https://doi.org/10.1016/j.frad.2014.09.004

[8] Jacques, C. and Dietemann, J.L. (2005) Imaging Features of Neurofibromatosis Type 1. Journal of Neuroradiology, 32, 180-197. https://doi.org/10.1016/S0150-9861(05)83136-0

[9] Hirbe, A.C. and Gutmann, D.H. (2014) Neurofibromatosis Type 1: A Multidisciplinary Approach to Care. The Lancet Neurology, 13, 834-843. https://doi.org/10.1016/S1474-4422(14)70063-8

[10] Lykissas, M.G., Schorry, E.K., Crawford, A.H., Gaines, S., Rieley, M. and Jain, V.V. (2013) Does the Presence of Dystrophic Features in Patients with Type 1 Neurofibromatosis and Spinal Deformities Increase the Risk of Surgery? Spine, 38, 1595-1601. https://doi.org/10.1097/BRS.0b013e31829a7779

[11] Ndoye, N., Kelani, A., Ba, M.C., Sokoum, I., Maïga, H.A., et al. (2011) Cervical Spinal Cord Slow Compression by a Neurofibroma in a Von Recklinghausen Disease: About 4 Cases. Journal Africain de Chirurgie, 1, 114-119.

[12] Rosai, J. (2011) Rosai J and Ackerman's Surgical Pathology. 10th Edition, Elsevier Saunders, Philadelphia. 